\title{
Decentralized Sensor Localization by Decision Fusion of RSSI and Mobility in Indoor Environments
}

\author{
Daniel Alshamaa, Farah Mourad-Chehade \\ Institut Charles Delaunay, ROSAS, LM2S \\ Université de Technologie de Troyes \\ UMR 6281, CNRS, Troyes, France \\ daniel.alshamaa@utt.fr, farah.chehade@utt.fr
}

\author{
Paul Honeine \\ LITIS lab \\ Université de Rouen Normandie \\ Rouen, France \\ paul.honeine@univ-rouen.fr
}

\begin{abstract}
Localization of sensors has become an essential issue in wireless networks. This paper presents a decentralized approach to localize sensors in indoor environments. The targeted area is partitioned into several sectors, each of which having a local calculator capable of emitting, receiving, and processing data. Each calculator runs a local localization algorithm, by investigating the belief functions theory for decision fusion of radio fingerprints, to estimate the sensors zones. The fusion of all calculators estimates, is combined with a mobility model to yield a final zone decision. The decentralized algorithm is described and evaluated against the state-of-the-art. Experimental results show the effectiveness of the proposed method in terms of localization accuracy, processing time, and robustness.

Index Terms-Decentralized architecture, decision fusion, localization, mobility, RSSI fingerprints.
\end{abstract}

\section{INTRODUCTION}

Wireless sensor networks (WSNs) have become an important research field [1]. Smart sensors are deployed in such networks and have the capability of processing data, and communicating with the environment. These sensors are highly communication-intensive systems [2], and have limited resources regarding processing, memory, and energy [3]. The network topology plays a vital role in WSNs in minimizing these resource constraints [4]. An efficient topology reduces the amount of communication required by the sensors to exchange information, and hence saves energy. A topology based on minimizing the distance between nodes for instance, reduces the probability of losing a message during communication. Moreover, a well-designed topology can also reduce radio interference, thus elongating the network lifetime [5].

Three main topologies have been proposed in WSNs [6], [7], [8]. On one hand, there exists the centralized topology where sensors acquire data measurements and transmit them to the fusion center [9]. In such a topology, the sensors are not required to carry out complex computations. Although it can achieve high quality processing, the centralized topology results in unnecessary energy costs due to the transmission of all measurements even if many are not needed [10]. On the other hand, the distributed topology treats equally all the sensors. The sensors exchange data with other sensors in their communication range. Since information processing is no longer limited to a single fusion center, the network is more robust to failures. However, developing relevant distributed algorithms remains a challenging issue. A compromise between the two is the decentralized topology, where the sensors are partitioned into several sectors, each having its own fusion center [11]. Information is exchanged between the sensors of each sector and transmitted to the local fusion center. The outputs of all fusion centers are combined to yield a final decision. Such a topology increases the scalability of the network, and reduces the energy consumption leading to a prolonged network lifetime [7].

Localization is an essential aspect in WSNs, since the knowledge of the sensor's location is critical to process the information originating from this sensor. To tackle the localization problem in indoor environments, researchers use various types of signals, such as ultra-wideband, WiFi, and Bluetooth [12], [13], [14]. One of the advantages of WiFi signals is that one can use the Access Points (APs) already installed in the building, with no additional hardware. The localization issue consists then in finding the location of a sensor node according to the $\mathrm{WiFi}$ signals that it collects from APs. Localization algorithms, such as Trilateration and connectivity-based methods, could be then applied. Such algorithms use a path loss model, which is practically inefficient in indoor environments [15], [16]. Alternatively, techniques that employ fingerprinting are widely implemented. They consist in collecting a database of exact reference locations, coupled to their corresponding WiFi signals strengths, received from the APs. The localization problem is then solved using this database and classification techniques [17], [18].

This paper investigates a decentralized architecture for zoning localization. Inspired by the decentralized topology, the proposed algorithm consists in partitioning the targeted area into several sectors, depending on the environment characteristics, and then assigning a calculator for each sector, that locally estimates the sensor's zone. Each calculator performs a local localization algorithm, using the belief functions theory (BFT) for decision fusion of radio fingerprints, to yield local zone estimates. The fusion of all calculators estimates is carried to assign evidence by an observation model. The latter is combined to a mobility model to make a final decision about the zone of the mobile sensor.

The rest of the paper is organized as follows. Section II describes the proposed decentralized localization approach. Section III demonstrates the experimental results, while section IV concludes the paper. 


\section{DECENTRALIZED LOCALIZATION}

In this section, the proposed decentralized localization method is explained. At first, we formulate the problem and describe the decentralized architecture, with the calculators' distribution. Next, we elucidate the local localization algorithm that will be run by each calculator. The fusion of calculator estimations is then discussed. At last, a mobility model is presented to enhance the overall localization accuracy.

\section{A. Description of the method}

The objective of the proposed algorithm is to determine the zone of a mobile sensor in a decentralized architecture. Suppose the targeted area is partitioned into $N_{C}$ sectors, each having its own calculator, thus obtaining $N_{C}$ calculators in total, denoted $C_{i}, i \in\left\{1, \ldots, N_{C}\right\}$. Let $Z_{j}, j \in J_{i}$, be the set of zones of the $i^{\text {th }}$ sector, $J_{i}$ being the set of indices of zones constituting sector $i$. Let $A P_{k}, k \in\left\{1, \ldots, N_{A P}\right\}$, be the APs installed in the network. Each AP broadcasts regularly WiFi signals in the network. At an offline phase, a sensor moves randomly in all zones to collect APs signals and measure their strengths, also called Received Signal Strength Indicators (RSSIs). Let $\rho_{j, k, r}, r \in\left\{1, \ldots, \ell_{j}\right\}$, be the set of $\ell_{j}$ RSSI measurements collected in an offline phase in the zone $Z_{j}$ with respect to $A P_{k}$. This leads to an offline database describing the variability of the RSSIs within and between the zones. Then in the online phase, a sensor to be localized measures a set of RSSIs from a certain number of APs and it broadcasts them in the network. Let $\rho_{\boldsymbol{t}} \in \mathbb{R}^{N_{A P}}$ be the vector of received RSSI measurements at time $t$. Since not all APs are detected at each instant, we denote $I_{A P, t} \in$ the set of indices of the APs whose signals are detected by the mobile node at time $t$ and $\rho_{k, t}, k \in I_{A P, t}$, their measured RSSIs. The vector $\rho_{t}$ is completed with zeros at positions where APs are not detected. The calculators within the sensor's neighborhood receive the RSSIs and use them with the offline database to assign evidence to each zone. Local estimations are then combined to reach a final decision of the sensor's zone. Formally speaking, the aim is to assign a level of confidence to each zone $\mathcal{C} f_{t}\left(Z_{j}\right), j \in J_{i}, i \in\left\{1, \ldots, N_{C}\right\}$, for any new online observation $\rho_{t}$ at the instant $t$.

To determine the number and the position of the calculators, an accuracy-based solution could be considered. One can measure the efficiency of the local localization algorithm by progressively increasing the number of zones and specifying an accuracy threshold. Once the overall accuracy falls below the threshold, no more zones are added, and thus sectors of approximately the same resulting number of zones or surface area are considered for each calculator. Calculators are then distributed in the same manner to cover the whole targeted area, positioning each calculator at the center of its corresponding sector. The calculators cover the zones of its sector at $100 \%$, which means that a message originating from any $Z_{j}, j \in J_{i}$, is certainly detected by $C_{i}$. To construct the decentralized architecture, we assign distinct sectors to each calculator, such that the zones constituting a certain sector are totally different than those constituting another one; i.e.

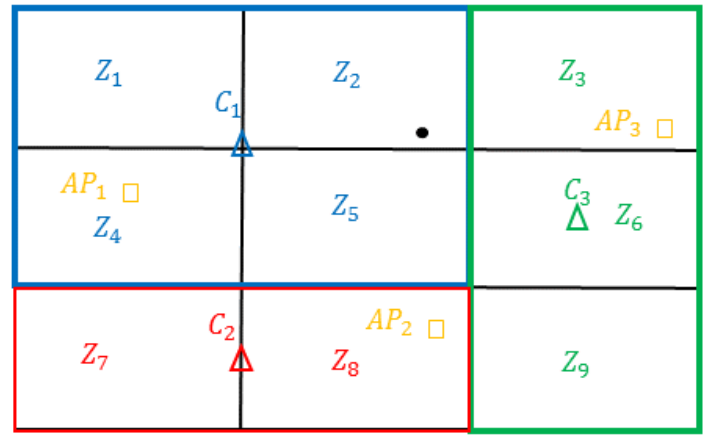

Fig. 1: Network architecture - $\triangle$ designates calculators, $\square$ designates Access Points, • designates mobile sensor.

$Z_{j} \neq Z_{j^{\prime}}, \quad \forall j \in J_{i}, j^{\prime} \in J_{i^{\prime}}, i, i^{\prime} \in\left\{1, \ldots, N_{C}\right\}, i \neq i^{\prime}$. An example of such an architecture is shown in Fig. 1, where calculator $C_{1}$ is responsible for the blue sector constituted of four zones, $C_{2}$ for the red sector of two zones, and $C_{3}$ for the green sector of three zones. In the following, $I_{C, t}$ would denote the set of indices of the calculators within the communication range of the sensor at time $t$.

\section{B. Local localization algorithm}

Each calculator detecting the sensor's message at time $t$, runs a local localization algorithm to localize sensors in its corresponding sector. We take advantage of the belief functions framework to assign evidence for each zone of the sector. The objective here is to determine a function $\mathcal{I}_{i, t}(\cdot): \mathbb{R}^{N_{A P}} \rightarrow[0,1]^{\left|J_{i}\right|}$ for each calculator $C_{i}$ such that $\mathcal{I}_{i, t}\left(\boldsymbol{\rho}_{t}\right)=m_{i, t}\left(Z_{j}\right), j \in J_{i}$ where $m_{i, t}\left(Z_{j}\right)$ is the evidence assigned to zone $Z_{j}$ due to new observation vector $\boldsymbol{\rho}_{t} \in \mathbb{R}^{N_{A P}}$.

In an offline phase, the collected RSSI measurements are fitted into parametric distributions according to a significance level $\alpha$. The significance level is a user-defined parameter to indicate the probability of rejecting the null hypothesis when it is true, and is set to less than $5 \%$. Once a new observation is carried for localization, the belief functions theory is used to generate the model $\mathcal{I}_{i, t}$. A detailed work on the usage of belief functions for zoning localization could be seen in [19]. Let $\mathcal{Z}_{i}$ be the set of all possible zones $Z_{j}, j \in J_{i}$ in sector $i$ and let $2^{\mathcal{Z}_{i}}$ be the set of all the non-empty subsets of $\mathcal{Z}_{i}$, i.e, $2^{\mathcal{Z}_{i}}=\left\{\emptyset,\left\{Z_{1}\right\}, \ldots, \mathcal{Z}_{i}\right\}$. A fundamental function of the BFT is the mass function, also defined as the basic belief assignment (BBA). A mass function $m_{A P_{k}, t}(\cdot)$ is a mapping from $2^{\mathcal{Z}_{i}}$ to the interval $[0,1]$, defined according to a certain source $A P_{k}, k \in\left\{1, \ldots, N_{A P}\right\}$. The mass $m_{A P_{k}, t}(A)$ given to $A \in 2^{\mathcal{Z}_{i}}$ stands for the proportion of evidence, brought by the source $A P_{k}$, saying that the measurement belongs to $A$ at instant $t$. In order to define the APs BBAs, all observations related to each AP belonging to a subset $A \in 2^{\mathcal{Z}_{i}}$ are fitted to a distribution $Q_{A P_{k}, A}$. Then, having an observation $\rho_{k, t}$ related to $A P_{k}, k \in I_{A P, t}$, the mass $m_{A P_{k}, t}(A)$ is calculated 
as follows,

$$
m_{A P_{k}, t}(A)=\frac{Q_{A P_{k}, A}\left(\rho_{k, t}\right)}{\sum_{A^{\prime} \in 2^{\mathcal{Z}}} Q_{A P_{k}, A^{\prime}}\left(\rho_{k, t}\right)}, \quad A \in 2^{\mathcal{Z}_{i}} .
$$

the evidence is then combined by aggregating the information coming from all the detected APs [20]. The mass functions can then be combined using the conjunctive rule of combination as follows,

$$
m_{\cap, i, t}(A)=\sum_{\substack{A^{(k)} \in 2^{z_{i}} \\ \cap_{k} A^{(k)}=A}} \prod_{k \in I_{A P, t}} m_{A P_{k}, t}\left(A^{(k)}\right),
$$

for all the subsets $A \in 2^{\mathcal{Z}_{i}}$, with $A^{(k)}$ is the subset $A$ with respect to the Access Point $A P_{k}$. An adequate notion of the BFT to attribute masses to singleton sets is the pignistic level [21]. It is defined as follows,

$$
\operatorname{Bet}_{i, t}(A)=\sum_{A \subseteq A^{\prime}} \frac{m_{\cap, i, t}\left(A^{\prime}\right)}{\left|A^{\prime}\right|},
$$

where $A$ is a singleton of $2^{\mathcal{Z}_{i}}$. The pignistic level is equivalent to the probability of having the observation belonging to the considered subset. Therefore, the evidence associated to singleton zones of sector $i$ could be deduced,

$$
m_{i, t}\left(Z_{j}\right)=\operatorname{Bet}_{i, t}\left(\left\{Z_{j}\right\}\right), j \in J_{i} .
$$

\section{Calculators fusion}

The described local localization algorithm is run by all calculators of $I_{C, t}$ to assign the masses $m_{i, t}\left(Z_{j}\right), j \in J_{i}$. Let $\eta_{i}$ be the strength of the signal received by calculator $C_{i}$ from the mobile sensor. If only one calculator receives the sensor's message, the mass assigned by this model will be identical to that assigned by the local calculator, and hence the mass attributed to each zone would be equal to the obtained mass, $m_{\mathbb{O}, t}\left(Z_{j}\right)=m_{i, t}\left(Z_{j}\right)$. We will call this an observation model, since the assigned masses are according to observed measurements. However, if more than one calculator detect the sensor's message, evidence is fused to reach a final estimation through the observation model. Since sectors are distinct, each zone has only one affiliated evidence. Thus, the fusion is all about weighting calculators decisions, followed by a final normalization phase on the whole set of zones. Since the strength of the signal decreases with the traveled distance, more confidence is given to calculators receiving stronger signals. The mass attributed to each zone by the observation model through the calculator fusion is then computed,

$$
m_{\mathbb{O}, t}\left(Z_{j}\right)=\frac{\frac{1}{w_{i}} \times m_{i, t}\left(Z_{j}\right)}{\sum_{p \in I_{C, t}} \sum_{q \in J_{p}} \frac{1}{w_{p}} \times m_{p, t}\left(Z_{q}\right)} .
$$

$w_{i}=\frac{\eta_{i}}{\sum_{x \in I_{C, t}} \eta_{x}}$ being the weight given to each calculator $C_{i}$. Eq. 5 computes masses for zones $Z_{j}, j \in J_{i}, i \in I_{C, t}$. The masses of zones outside these sectors are null, since an undetected message by a calculator means for sure that the sensor is not in any zone of its corresponding sector.
In addition to its simplicity, an important advantage of the described architecture and its corresponding calculator fusion approach is its scalability. Any expansion of the targeted area could easily be treated by adding the new calculators to the expanded area without any modification on the already existing architecture. Moreover, it reduces energy consumption, as mobile sensors are required to send information to calculators in their range. It is also more robust, since the existence of several calculators renders the network more resistant to failures that are fatal in the case of a centralized approach.

\section{Mobility}

To enhance the accuracy of the localization algorithm, we benefit of the notion of mobility to add as another source of information. Let $v_{\max }$ be the maximum speed of the mobile sensor in the target area, $\Delta t_{l o c}$ the localization algorithm execution time, and $d_{x y}$ the minimal geographical distance between the two zones $Z_{x}$ and $Z_{y}$. The maximum distance that the sensor can travel is then deduced $d_{\max }=v_{\max } \times$ $\Delta t_{l o c}$. Let $p_{x y}, x, y \in\left\{1, \ldots, N_{Z}\right\}$, denote the coefficient of transition from zone $Z_{x}$ to zone $Z_{y}$ within $\Delta t_{l o c}$. Then,

$$
p_{x y}= \begin{cases}0, & \text { if } d_{\max }<d_{x y} \\ 1, & \text { if } d_{\max } \geq d_{x y} .\end{cases}
$$

As we have indicated in section II.A, the objective is to assign a confidence level for all zones $\mathcal{C} f_{t}\left(Z_{j}\right), j \in J_{i}, i \in$ $\left\{1, \ldots, N_{C}\right\}$. This confidence level will be the final decision, which is the fusion of all the evidence from observation and mobility models. The aim of the mobility model is to use the confidence at a previous instant to know where the sensor might be at present.

Being in a zone $Z_{x}$ at instant $t-1$, the sensor could be at instant $t$ at any zone $y \in\left\{1, \ldots, N_{Z}\right\}$ of the ones having $p_{x y}=1$. These zones are called the following zones of $Z_{x}$. The confidence of $Z_{x}$ at time $t-1$ is then propagated to time $t$ by distributing it equally to its following zones, each one would be having $\frac{\mathcal{C} f_{t-1}\left(Z_{i}\right)}{\sum_{y} p_{x y}}$. The mobility evidence given to a zone $Z_{j}$ at time $t$ is the aggregation of all evidence deduced from its preceding ones having $p_{x j}=1, \forall x \in J_{i}, i \in I_{C, t-1}$. Only zones that could have an observation evidence at time $t$ are considered. This leads to a mobility mass at time $t$,

$$
m_{\mathbb{M}, t}\left(Z_{j}\right)=\sum_{x \in J_{i}, i \in I_{C, t-1}} p_{x j} \times \frac{\mathcal{C} f_{t-1}\left(Z_{x}\right)}{\sum_{y \in J_{i}, i \in I_{C, t}} p_{x y}} .
$$

\section{E. Global decision}

The evidence obtained through the calculators fusion is combined with that obtained by the mobility model, to yield a final confidence level to be assigned to all zones,

$$
\mathcal{C} f_{t}\left(Z_{j}\right)=\frac{m_{\mathbb{O}, t}\left(Z_{j}\right) \times m_{\mathbb{M}, t}\left(Z_{j}\right)}{\sum_{\chi \in J_{i}, i \in I_{C, t}} m_{\mathbb{O}, t}\left(Z_{\chi}\right) \times m_{\mathbb{M}, t}\left(Z_{\chi}\right)} .
$$

The algorithm then selects the zone $\hat{Z}_{j, t}$ having the highest assigned level of confidence at instant $t$,

$$
\hat{Z}_{j, t}=\arg \max _{j \in J_{i}, i \in I_{C, t}} \mathcal{C} f_{t}\left(Z_{j}\right) \text {. }
$$




\section{EXPERIMENTS}

Experiments are realized in the statistical and operational research department of the University of Technology of Troyes, France. The targeted area is approximately $500 \mathrm{~m}^{2}$ and is constituted of 21 zones. The sensors scan the network and distinguish APs through their MAC addresses. They then measure the RSSIs and send the information that will be detected by any set of the 6 available calculators at time $t$. It is noted that 23 different AP networks could be detected and hence are used as sources of information. To apply the proposed method, the decentralized architecture is constructed as described in II.A. Each calculator runs the local localization algorithm to assign evidence to each zone of its sector. A set of 50 RSSI measurements is taken in each zone of which 30 were used to fit the distributions at a 0.02 significance level, and 20 for test in the online phase. The assumed maximum speed is $1.5 \mathrm{~m} / \mathrm{s}$. An estimation is said to be correct if the algorithm assigns the highest confidence to the zone where the sensor actually resides.

Table I shows the performance of the proposed method and compares it to a belief functions centralized approach (BCA) presented in [14], and to a hierarchical centralized approach (HCA) presented in [19]. The BCA uses the BFT to fuse all evidence using one calculator, while the HCA creates a hierarchy of clusters from the original zones using divergence similarity. A decentralized version of HCA would not be applicable in this case as large number of zones are required for it to be effective. To evaluate the robustness of the various techniques, the accuracy is recorded as a function of the number of failing fusion centers or calculators. For each new observation, a random set of calculators is supposed to have failed. The overall accuracy is then determined by the average of the obtained accuracy on all observations with respect to $x$ failing calculators, $x=\{0, \ldots, 6\}$. As the table shows, the proposed method achieves a better overall accuracy as compared to the centralized approaches. Moreover, both BCA and HCA would not be able to perform any localization if one or more calculators fail. In fact, there is only one calculator in such architectures and hence its failure means the failure of the whole network. On the other hand, the decentralized approaches can still manage to localize the sensors upon failure of a certain number of calculators, even though the overall accuracy degrades. This robustness is a clear advantage of the decentralized technique as it allows localization upon calculators failure, which could not be achieved by the centralized methods.

In this paragraph, the proposed method is compared to well-known localization techniques. In [22], the authors present a weighted $k$-nearest neighbors algorithm (WKNN) for indoor localization. To estimate the position of the sensor, the received measurement is compared with the elements in the fingerprint database using Euclidean distances. A set of $k$ smallest Euclidean distances is selected and the $k$-nearest neighbors algorithm is then applied. The algorithm averages the coordinates of the $k$-nearest neighbors of the sensor,
TABLE I: Influence of fusion center failure on the overall accuracy of the decentralized and centralized approaches.

\begin{tabular}{lrrrrrrr}
\hline \multirow{2}{*}{ Technique } & \multicolumn{6}{c}{ Number of failed fusion centers } \\
\cline { 2 - 8 } & 0 & 1 & 2 & 3 & 4 & 5 & 6 \\
\hline BCA & 78.61 & - & - & - & - & - & - \\
HCA & 87.77 & - & - & - & - & - & - \\
Proposed & 90.47 & 81.67 & 70.28 & 58.33 & 29.72 & 22.50 & - \\
\hline
\end{tabular}

TABLE II: Comparison between various localization techniques in terms of accuracy and processing time.

\begin{tabular}{lrr}
\hline Technique & accuracy $(\%)$ & processing \\
\hline WKNN & 83.88 & 0.1289 \\
connectivity & 86.67 & 0.1338 \\
NN & 84.17 & 0.1866 \\
SVM & 85.55 & 0.1859 \\
BCA & 78.61 & 0.1147 \\
HCA & 87.77 & 0.2508 \\
Proposed & 90.56 & 0.0977 \\
\hline
\end{tabular}

weighting each distance by a factor previously specified according to a mathematical model, to give its location estimate. In [23], a connectivity-based localization algorithm has been proposed. Connectivity-based algorithms are range free, which means that they do not rely on collected measurements. The sensor's location is given as the intersection of the ranges of the APs detected by the sensor. On the other hand, conventional classification techniques such neural network (NN) [24] and Support Vector Machines (SVM) [18] could be applied. Table II compares the overall accuracy and the localization processing time of the various described techniques. As the table shows, the proposed method outperforms other state-ofthe-art localization techniques in terms of overall accuracy and processing time. The proposed method gains advantage of the simplicity of the local localization algorithm, which results in a relatively low processing time.

An important factor to measure also is the dependency of the localization method on the number of APs. A method that requires a high density of APs is not favored for instance, as it is practically unfeasible in most of the cases due to the unavailability of sufficient APs in the network, or due to the installation cost. Table III shows the overall accuracy as a function of the number of available APs. It is noted that both WKNN and connectivity methods are highly sensitive to the density of APs, while the proposed method is less sensitive at the level with a decrease of $8.3 \%$ in overall accuracy, upon a decrease in the number of detected APs from 23 to 5 .

As it could be concluded from the aforementioned results, the proposed decentralized localization algorithm is more robust and less complex than centralized approaches. It also outperforms other well-known techniques in the domain at the level of accuracy and online processing time. In addition, 
TABLE III: Influence of APs density on the overall accuracy of the decentralized and centralized approaches.

\begin{tabular}{lrrrr}
\hline \multirow{2}{*}{ Technique } & \multicolumn{4}{c}{ Number of detected APs } \\
\cline { 2 - 5 } & 5 & 10 & 15 & 23 \\
\hline WKNN & 67.22 & 72.78 & 74.17 & 83.88 \\
connectivity & 65.56 & 69.44 & 76.67 & 84.17 \\
NN & 77.78 & 80.00 & 81.39 & 84.72 \\
SVM & 78.61 & 80.83 & 82.78 & 85.55 \\
BCA & 74.17 & 76.94 & 77.50 & 78.61 \\
HCA & 80.56 & 83.33 & 84.44 & 87.77 \\
Proposed & 82.22 & 83.33 & 85.27 & 90.56 \\
\hline
\end{tabular}

it is less sensitive to the density of APs in the network, as localization is to be done locally on small surfaces without the need of many APs to achieve high accuracy.

\section{CONCLusion}

This paper presents a decentralized approach for localization of sensors in indoor wireless networks. At first, a decentralized architecture is constructed, partitioning the target area into sectors, each having its own calculator. A local localization algorithm is then run by each calculator using the belief functions theory. Afterwards, the calculators estimations are fused, resulting in an observation model. The proposed algorithm then uses the mobility of sensors to assign another type of evidence. Levels of confidence are attributed to zones by fusing evidence from both observation and mobility models. Experiments are conducted to evaluate the performance of the decentralized approach, and compare it against a centralized one. In addition, the accuracy, complexity, robustness, and the influence of detected Access Points on the localization algorithm are studied in comparison to wellknown techniques in the domain. Future work will focus on kernel-based models for cases where parametric distributions fail to represent the RSSI. In addition, advanced mobility models such as hidden Markov models will be studied to enhance the overall localization algorithm.

\section{ACKNOWLEDGEMENT}

The authors would like to thank the European Regional Development Fund and the Grand Est Region in France for funding this work, project LIPAD.

\section{REFERENCES}

[1] H. Peng, S. Si, M. K. Awad, N. Zhang, H. Zhao, and X. S. Shen, "Toward energy-efficient and robust large-scale wsns: a scale-free network approach," IEEE Journal on Selected Areas in Communications, vol. 34, no. 12, pp. 4035-4047, 2016.

[2] B. Zhou, S. Yang, T. Sun, and K. T. Grattan, "A novel wireless mobile platform to locate and gather data from optical fiber sensorsintegrated into a wsn," IEEE Sensors Journal, vol. 15, no. 6, pp. 3615-3621, 2015.

[3] M. Nikolov and Z. J. Haas, "Encoded sensing for energy efficient wireless sensor networks," IEEE Sensors Journal, 2017.
[4] L. Chen, G. Carpenter, S. Greenberg, J. Frolik, and X. S. Wang, "An implementation of decentralized consensus building in sensor networks," IEEE Sensors Journal, vol. 11, no. 3, pp. 667-675, 2011.

[5] R. Velmani and B. Kaarthick, "An efficient cluster-tree based data collection scheme for large mobile wireless sensor networks," IEEE sensors journal, vol. 15, no. 4, pp. 2377-2390, 2015.

[6] J. Yan, H. Liu, W. Pu, and Z. Bao, "Decentralized 3-d target tracking in asynchronous 2-d radar network: Algorithm and performance evaluation," IEEE Sensors Journal, vol. 17, no. 3, pp. 823-833, 2017.

[7] M. Üney, B. Mulgrew, and D. E. Clark, "A cooperative approach to sensor localisation in distributed fusion networks," IEEE Transactions on Signal Processing, vol. 64, no. 5, pp. 1187-1199, 2016.

[8] J. Cota-Ruiz, P. Rivas-Perea, E. Sifuentes, and R. Gonzalez-Landaeta, "A recursive shortest path routing algorithm with application for wireless sensor network localization," IEEE Sensors Journal, vol. 16, no. 11, pp. 4631-4637, 2016.

[9] H. Talebi and A. A. Hemmatyar, "Asynchronous track-to-track fusion by direct estimation of time of sample in sensor networks," IEEE sensors journal, vol. 14, no. 1, pp. 210-217, 2014.

[10] Q. Mamun, "A qualitative comparison of different logical topologies for wireless sensor networks," Sensors, vol. 12, no. 11, pp. 14887-14913, 2012.

[11] S. Mahfouz, F. Mourad-Chehade, P. Honeine, J. Farah, and H. Snoussi, "Decentralized localization using fingerprinting and kernel methods inwireless sensor networks," in Signal Processing Conference (EUSIPCO), 2013 Proceedings of the 21st European, pp. 1-5, IEEE, 2013.

[12] H. Liu, H. Darabi, P. Banerjee, and J. Liu, "Survey of wireless indoor positioning techniques and systems," Systems, Man, and Cybernetics, Part C: Applications and Reviews, IEEE Transactions on, vol. 37, pp. 1067-1080, Nov 2007.

[13] A. Disha, "A comparative analysis on indoor positioning techniques and systems," International Journal of Engineering Research and Applications, vol. 3, pp. 1790-1796, 2013.

[14] D. Alshamaa, F. Mourad-Chehade, and P. Honeine, "Zoning-based localization in indoor sensor networks using belief functions theory," in Signal Processing Advances in Wireless Communications (SPAWC), 2016 IEEE 17th International Workshop on, pp. 1-5, IEEE, 2016.

[15] J. Sangthong, P. Dokpikul, and S. Promwong, "Indoor positioning based on IEEE 802.15. 4a standard using trilateration technique and UWB signal," in Proceedings in Progress in Electromagnetics Research Symposium, 2012.

[16] J. M. Pak, C. K. Ahn, Y. Shmaliy, and M. T. Lim, "Improving reliability of particle filter-based localization in wireless sensor networks via hybrid particle/FIR filtering," Industrial Informatics, IEEE Transactions on, vol. 11, pp. 1089-1098, Oct 2015.

[17] D. Alshamaa, F. M. Chehade, and P. Honeine, "A hierarchical classification method using belief functions," Signal Processing, vol. 148, pp. $68-77,2018$.

[18] L. Zhang, Y. Li, Y. Gu, and W. Yang, "An efficient machine learning approach for indoor localization," China Communications, vol. 14, no. 11, pp. 141-150, 2017.

[19] D. Alshamaa, F. Mourad-Chehade, and P. Honeine, "Tracking of mobile sensors using belief functions in indoor wireless networks," IEEE Sensors Journal, vol. 18, no. 1, pp. 310-319, 2017.

[20] M. Kurdej and V. Cherfaoui, "Conservative, proportional and optimistic contextual discounting in the belief functions theory," in Information Fusion (FUSION), 2013 16th International Conference on, pp. 20122018, IEEE, 2013.

[21] P. Smets, "Belief functions: the disjunctive rule of combination and the generalized Bayesian theorem," International Journal of approximate reasoning, vol. 9, no. 1, pp. 1-35, 1993.

[22] H. Koyuncu and S. H. Yang, "A 2d positioning system using wsns in indoor environment," International Journal of Electrical and Computer Sciences, vol. 11, no. 3, pp. 70-77, 2011.

[23] Y. Shang, W. Rumi, Y. Zhang, and M. Fromherz, "Localization from connectivity in sensor networks," IEEE Transactions on parallel and distributed systems, vol. 15, no. 11, pp. 961-974, 2004.

[24] H. Dai, W.-h. Ying, and J. Xu, "Multi-layer neural network for received signal strength-based indoor localisation," IET Communications, vol. 10 , no. 6 , pp. 717-723, 2016. 